Pure and Applied Mathematics Quarterly

Volume 6, Number 3

(Special Issue: In honor of

Joseph J. Kohn, Part 1 of 2$)$

$677-692,2010$

\title{
The Heat Kernel for Gravitational Potential Operators in One and Two Variables
}

\author{
Ovidiu Calin* and Der-Chen Chang $^{\dagger}$
}

Dedicated to Professor J.J.Kohn on the occasion of his 75th birthday and retirement

Abstract: In this article we find closed form formulas for the heat kernels of the operators $\frac{1}{2} \partial_{x}^{2}+\frac{\lambda}{x^{2}}$ and $\frac{1}{2}\left(\partial_{x}^{2}+\partial_{y}^{2}\right)-\frac{\lambda^{2}}{x^{2}+y^{2}}$. We are using a geometric method and the expansion over eigenvalues method.

Keywords: heat equation, Euler-Lagrange equation, Hamiltonian, Lagrangian, Van Vleck determinant, Laguerre polynomials.

\section{Introduction}

In the first part of this paper we shall obtain the heat kernel for the operator

$$
\mathbb{L}=\frac{1}{2} \partial_{x}^{2}+\frac{\lambda}{x^{2}}, \quad \lambda \in \mathbb{R},
$$

involving a geometric technique presented in [1]. An approach using path integrals can be found in [4]. An extension to a propagator involving time-dependent

Received December 12, 2007.

MS Classification (2000): Primary: 53C17; Secondary: 34K10, 35H20

*The first author is partially supported by NSF grant \#0631541.

${ }^{\dagger}$ The second author is partially supported by a Hong Kong RGC competitive earmarked research grant \#600607 and a competitive research grant at Georgetown University. 
harmonic oscillator was done by using path integrals in [5]. The authors of this paper have been inspired to work on this problem after getting acquainted with the problem from [6].

This operator owes its physical significance to its relation to the two body problem. Consider two unit mass particles described by the coordinates $\xi_{1}$ and $\xi_{2}$ on the real line with an interaction which varies as the inverse square of the distance between the particles. The problem is characterized by a Lagrangian which is the difference between the kinetic energy and the gravitational potential

$$
L(\dot{x}, x)=\frac{1}{2}\left(\dot{\xi}_{1}^{2}+\dot{\xi}_{2}^{2}\right)-\frac{2 \lambda}{\left(\xi_{1}-\xi_{2}\right)^{2}},
$$

where $\lambda>0$ is the strength of the interaction.

With the change of coordinates

$$
x=\frac{\xi_{1}-\xi_{2}}{\sqrt{2}}, \quad y=\frac{\xi_{1}+\xi_{2}}{2}
$$

the Lagrangian (1.2) becomes the following sum of two Lagrangians

$$
L(x, y, \dot{x}, \dot{y})=\dot{y}^{2}+\left(\frac{1}{2} \dot{x}^{2}-\frac{\lambda}{x^{2}}\right)=L_{0}+L_{1}
$$

The momenta associated with the coordinates $x$ and $y$ are

$$
p_{x}=\frac{\partial L}{\partial \dot{x}}=\frac{\partial L_{1}}{\partial \dot{x}}=\dot{x}, \quad p_{y}=\frac{\partial L}{\partial \dot{y}}=\frac{\partial L_{0}}{\partial \dot{x}}=2 \dot{y} .
$$

Then the associated Hamiltonian is obtained by applying the Legendre transform on the Lagrangian $L$

$$
\begin{aligned}
H\left(p_{x}, p_{y}, x, y\right) & =p_{x} \dot{x}+p_{y} \dot{y}-L=\left(p_{y} \dot{y}-L_{0}\right)+\left(p_{x} \dot{x}-L_{1}\right) \\
& =H_{0}\left(p_{y}, y\right)+H_{1}\left(p_{x}, x\right)
\end{aligned}
$$

with

$$
H_{0}\left(p_{y}, y\right)=\frac{p_{y}^{2}}{4}, \quad H_{1}\left(p_{x}, x\right)=\frac{1}{2} p_{x}^{2}+\frac{\lambda}{x^{2}}
$$


Quantizing the Hamiltonian $H=H_{0}+H_{1}$, i.e., replacing $p_{x} \rightarrow \partial_{x}$ and $p_{y} \rightarrow \partial_{y}$ yields the following operator

$$
\mathbb{P}=\frac{1}{4} \partial_{y}^{2}+\frac{1}{2} \partial_{x}^{2}+\frac{\lambda}{x^{2}}
$$

Separating the variables, the heat kernel of $\mathbb{P}$ can be written as

$$
\begin{aligned}
e^{t \mathbb{P}} \delta_{\left(x_{0}, y_{0}\right)} & =e^{t \frac{1}{4} \partial_{y}^{2}+t\left(\frac{1}{2} \partial_{x}^{2}+\frac{\lambda}{x^{2}}\right)} \delta_{\left(x_{0}, y_{0}\right)} \\
& =e^{t \frac{1}{4} \partial_{y}^{2}} \delta_{y_{0}} \otimes e^{t\left(\frac{1}{2} \partial_{x}^{2}+\frac{\lambda}{x^{2}}\right)} \delta_{x_{0}} \\
& =\frac{1}{\sqrt{16 \pi t}} e^{-\frac{\left(y-y_{0}\right)^{2}}{16 t}} \otimes e^{t\left(\frac{1}{2} \partial_{x}^{2}+\frac{\lambda}{x^{2}}\right)} \delta_{x_{0}}, \quad t>0 .
\end{aligned}
$$

We need to compute the second term of the above convolution which is the heat kernel of the operator (1.1). We recall in the following section a geometric method for computing heat kernels.

In the second part of this paper we shall treat the heat kernel problem for the operator

$$
\mathbb{L}=\frac{1}{2}\left(\partial_{x}^{2}+\partial_{y}^{2}\right)-\frac{\lambda^{2}}{x^{2}+y^{2}}
$$

The method is using Laguerre polynomials and the expansion over the eigenfunctions. The exact summation is given by the Hille-Hardy's formula, see [2].

\section{Heat kernels for some operators with potential}

The following variational method is related to the geometry generated by the differential operator. In the following we shall briefly describe the method. This shows the interrelation between the geometry of an operator and its heat kernel.

In this section the differential operators will be of the form

$$
\mathbb{L}=\frac{1}{2}{\frac{d^{2}}{d x}}^{2}+U(x)
$$

with $U(x)$ at most quadratic. We associate the Hamiltonian function

$$
H(p, x)=\frac{1}{2} p^{2}+U(x) .
$$


Hamilton's equations are

$$
\begin{aligned}
& \dot{x}=H_{p}=p, \\
& \dot{p}=-H_{x}=-U^{\prime}(x), \text { and hence } \ddot{x}=\dot{p}=-U^{\prime}(x) .
\end{aligned}
$$

For any two given points $x_{0}$ and $x$, the geodesic joining them is obtained solving the equation

$$
\left\{\begin{array}{l}
\ddot{x}=-U^{\prime}(x), \\
x(0)=x_{0}, \\
x(t)=x .
\end{array}\right.
$$

We shall assume that $U(x)$ is such that the system (2.7) has a unique solution $x(s)$. The classical action between $x_{0}$ and $x$ in time $t$ is obtained integrating the Lagrangian $L=p \dot{x}-H=\frac{1}{2} \dot{x}^{2}-U(x)$ along the above geodesic $x(s)$

$$
S_{c l}\left(x_{0}, x ; t\right)=\int_{0}^{t} L(\dot{x}(s), x(s)) d s=\int_{0}^{t} \frac{1}{2} \dot{x}^{2}(s)-U(x(s)) d s .
$$

With these notations the heat kernel of the operator (2.5), which is a path integral, is given by the formula

$$
K\left(x_{0}, x ; t\right)=V(t) e^{-S_{c l}\left(x_{0}, x ; t\right)}
$$

If the Lagrangian is at most quadratic, then $V(t)=\sqrt{\operatorname{det}\left(-\frac{1}{2 \pi} \frac{\partial^{2} S_{c l}}{\partial x \partial x_{0}}\right)}$ is the Van Vleck determinant. Otherwise, the function $V$ depends on $t, x_{0}$ and $x$ and satisfies a transport equation, see section 4 .

\section{Finding the classical action}

The geodesic joining the points $x_{0}$ and $x$ in time $t$ satisfies the Euler-Lagrange equation associated with the Lagrangian $L_{1}$

$$
\begin{aligned}
\ddot{x} & =\frac{2 \lambda}{x^{3}} \\
x(0) & =x_{0}, \quad x(t)=x .
\end{aligned}
$$


Since the regions $\{x<0\}$ and $\{x>0\}$ are separated, in order to have connectivity, we have to assume that either $x_{0}, x>0$ or $x_{0}, x<0$. We can show that the energy is a first integral of motion, so

$$
\frac{1}{2} \dot{x}^{2}(s)+\frac{\lambda}{x^{2}(s)}=E, \quad s \in[0, t]
$$

Under the assumption $x_{0}, x>0$ the above relation becomes

$$
\dot{x}(s) x(s)=\sqrt{2 E x^{2}(s)-2 \lambda} .
$$

Let $u(s)=x^{2}(s), u_{0}=x_{0}^{2}, u_{t}=x^{2}(t)=x^{2}$. Then $u(s)$ verifies the ODE

$$
\begin{aligned}
\dot{u} & =2 \sqrt{2 E u-2 \lambda} \\
u(0) & =u_{0}, \quad u(t)=u_{t} .
\end{aligned}
$$

We note that $\dot{u}>0$, so the right side must have positive sign. Integrating yields

$$
\begin{gathered}
\int_{u_{0}}^{u_{t}} \frac{d u}{\sqrt{2 E u-2 \lambda}}=2 t \Longleftrightarrow \\
\sqrt{2 E u_{t}-2 \lambda}-\sqrt{2 E u_{0}-2 \lambda}=2 E t .
\end{gathered}
$$

Eliminating the square roots we obtain

$$
\left(\left(u_{0}+u_{t}\right)-2 E t^{2}\right)^{2}=4\left(u_{0} u_{t}-2 \lambda t^{2}\right),
$$

where we assume the condition

$$
\lambda<\frac{x_{0}^{2} x^{2}}{2 t^{2}}
$$

Solving for $E$ in (3.9) yields

$$
E=\frac{x_{0}^{2}+x^{2}}{2 t^{2}}-\frac{\sqrt{x_{0}^{2} x^{2}-2 \lambda t^{2}}}{t^{2}} .
$$

The classical action $S_{c l}$ satisfies the following Hamilton-Jacobi equation

$$
\partial_{t} S_{c l}=-E,
$$


with $E$ given by (3.10). We can write $S_{c l}=S_{0}+S_{1}$, where

$$
\begin{aligned}
\partial_{t} S_{0} & =-\frac{x_{0}^{2}+x^{2}}{2 t^{2}} \Longrightarrow S_{0}=\frac{x_{0}^{2}+x^{2}}{2 t} \\
\partial_{t} S_{1} & =\frac{\sqrt{x_{0}^{2} x^{2}-2 \lambda t^{2}}}{t^{2}}
\end{aligned}
$$

We shall solve (3.12) as a homogeneous equation. Let $\tau=\frac{t}{x_{0} x}$, and define $S_{2}(\tau)=S_{1}\left(\frac{t}{x_{0} x}\right)$. Then

$$
\frac{d}{d \tau} S_{2}(\tau)=\frac{\sqrt{1-2 \lambda \tau^{2}}}{\tau^{2}} .
$$

With the substitution $\tau=\frac{1}{\sqrt{2 \lambda}} \sin \phi$, integrating yields

$$
\begin{aligned}
S_{2}(\tau) & =\int \frac{\sqrt{1-2 \lambda \tau^{2}}}{\tau^{2}} d \tau=\sqrt{2 \lambda} \int \cot ^{2} \phi d \phi \\
& =\sqrt{2 \lambda} \int\left(-1-\cot ^{\prime} \phi\right) d \phi=-\sqrt{2 \lambda}(\phi+\cot \phi) \\
& =-\sqrt{2 \lambda}\left\{\sin ^{-1}(\sqrt{2 \lambda} \tau)+\frac{\sqrt{1-2 \lambda \tau^{2}}}{\sqrt{2 \lambda} \tau}\right\}
\end{aligned}
$$

and hence

$$
S_{1}\left(x_{0}, x, \tau\right)=\frac{-2 \sqrt{x_{0}^{2} x^{2}-\lambda t^{2}}}{2 t}-\sqrt{2 \lambda} \sin ^{-1}\left(\sqrt{2 \lambda} \frac{t}{x_{0} x}\right) .
$$

From (3.13) and (3.11) we obtain the classical action

$$
S_{c l}\left(x_{0}, x, t\right)=\frac{x_{0}^{2}+x^{2}}{2 t}-\frac{2 \sqrt{x_{0}^{2} x^{2}-\lambda t^{2}}}{2 t}-\sqrt{2 \lambda} \sin ^{-1}\left(\sqrt{2 \lambda} \frac{t}{x_{0} x}\right) .
$$

By the general theory, or by a direct computation, the classical action $S_{c l}$ satisfies the Hamilton-Jacobi equation

$$
\partial_{t} S_{c l}+\frac{1}{2}\left(\partial_{x} S_{c l}\right)^{2}+\frac{\lambda}{x^{2}}=0
$$

We also note that for $\lambda \rightarrow 0$ we obtain the Euclidean action

$$
S_{c l}\left(x_{0}, x, t\right)=\frac{\left(x-x_{0}\right)^{2}}{2 t} .
$$




\section{The transport equation}

We shall assume that the heat kernel of $\mathbb{L}$ is of the type

$$
\mathcal{K}\left(x_{0}, x, t\right)=V(x, t) e^{-S\left(x_{0}, x, t\right)} .
$$

Then a computation shows

$$
\begin{aligned}
& \partial_{t} \mathcal{K}=e^{-S}\left(\partial_{t} V-V \partial_{t} S\right) \\
& \partial_{x}^{2} \mathcal{K}=e^{-S}\left(\partial_{x}^{2} V-2 \partial_{x} V \partial_{x} S+V\left(\partial_{x} S\right)^{2}-V\left(\partial_{x}^{2} S\right)\right)
\end{aligned}
$$

and hence

$$
\begin{aligned}
\left(\partial_{t}-\mathbb{L}\right) \mathcal{K}= & \left(\partial_{t}-\frac{1}{2} \partial_{x}^{2}-\frac{\lambda}{x^{2}}\right) \mathcal{K} \\
= & e^{-S}\left\{-V[\underbrace{\partial_{t} S+\frac{1}{2}\left(\partial_{x} S\right)^{2}+\frac{\lambda}{x^{2}}}_{=0}](b y)\right. \\
& \left.+\partial_{t} V-\frac{1}{2} \partial_{x}^{2} V+\partial_{x} V \partial_{x} S+\frac{1}{2} V \partial_{x}^{2} S\right\} .
\end{aligned}
$$

We shall ask $V$ to satisfy the following transport equation

$$
\partial_{t} V-\frac{1}{2} \partial_{x}^{2} V+\partial_{x} V \partial_{x} S+\frac{1}{2} V \partial_{x}^{2} S=0 .
$$

The equation (4.16) might be hard to solve since the action $S$ and its derivatives are complicated. In the following we shall consider a shortcut for these computations. We note that the action $S=S_{0}+S_{1}$, where the term $S_{1}$ is a function of $\frac{x_{0} x}{t}$. Then

$$
e^{-S}=W\left(\frac{x_{0} x}{t}\right) e^{-\frac{x_{0}^{2}+x^{2}}{2 t}},
$$

for some function $W$. Then it makes sense to look now for a heat kernel of the type

$$
\mathcal{K}\left(x_{0}, x, t\right)=V(x, t) e^{-S_{0}}=V(x, t) e^{-\frac{x_{0}^{2}+x^{2}}{2 t}},
$$

where $V(x, t)=\frac{1}{\sqrt{t}} Z\left(\frac{x_{0} x}{t}\right)$, for some function $Z$, satisfies the following extended transport equation 


$$
\partial_{t} V-\frac{1}{2} \partial_{x}^{2} V+\partial_{x} V \partial_{x} S_{0}+\frac{1}{2} V\left(\partial_{x}^{2} S_{0}\right)-V\left[\partial_{t} S_{0}+\frac{1}{2}\left(\partial_{x} S_{0}\right)^{2}+\frac{\lambda}{x^{2}}\right]=0
$$
have

In the following we shall solve the equation (4.17). Let $\tau=\frac{x_{0} x}{t}$. Then we

$$
\begin{aligned}
V & =\frac{1}{\sqrt{t}} Z(\tau) \\
\partial_{t} V & =-\frac{1}{t \sqrt{t}}\left(\frac{1}{2} Z(\tau)+\tau Z^{\prime}(\tau)\right) \\
\partial_{x} V & =\frac{1}{t \sqrt{t}} Z^{\prime}(\tau) x_{0} \\
\partial_{x}^{2} V & =\frac{1}{t \sqrt{t}} Z^{\prime \prime}(\tau) \frac{x_{0}^{2}}{t} .
\end{aligned}
$$

Since

$$
\partial_{t} S=-\frac{x_{0}^{2}+x^{2}}{2 t^{2}}, \quad \partial_{x} S=\frac{x}{t}, \quad \partial_{x}^{2} S=\frac{1}{t},
$$

the equation (4.17) becomes after cancelations

$$
-\frac{1}{2} \frac{1}{\sqrt{t}} Z^{\prime \prime}(\tau)\left(\frac{x_{0}}{t}\right)^{2}-\frac{1}{\sqrt{t}} Z(\tau)\left[\frac{\lambda}{x^{2}}-\frac{1}{2}\left(\frac{x_{0}}{t}\right)^{2}\right]=0 .
$$

Multiplying by $-2 x^{2} t^{\frac{1}{2}}$ yields

$$
\tau^{2} Z^{\prime \prime}(\tau)+Z(\tau)\left[2 \lambda-\tau^{2}\right]=0
$$

Let $U(\tau)=\tau^{-\frac{1}{2}} Z(\tau)$. A computation shows

$$
\begin{aligned}
\tau^{2} U^{\prime \prime}(\tau) & =\frac{3}{4} \frac{1}{\sqrt{\tau}} Z(\tau)-\tau^{\frac{1}{2}} Z^{\prime}(\tau)+\tau \sqrt{\tau} Z^{\prime \prime}(\tau) \\
\tau U^{\prime}(\tau) & =-\frac{1}{2} \frac{1}{\sqrt{\tau}} Z(\tau)+\sqrt{\tau} Z^{\prime}(\tau),
\end{aligned}
$$

and using (4.18) we have

$$
\begin{aligned}
\tau^{2} U^{\prime \prime}(\tau)+\tau U^{\prime}(\tau) & =\frac{1}{\sqrt{\tau}}\left(\tau^{2} Z^{\prime \prime}(\tau)+\frac{1}{4} Z(\tau)\right) \\
& =\frac{1}{\sqrt{\tau}}\left(Z(\tau)\left(\tau^{2}-2 \lambda\right)+\frac{1}{4} Z(\tau)\right) \\
& =U(\tau)\left(\tau^{2}-2 \lambda+\frac{1}{4}\right) .
\end{aligned}
$$


Hence $U(\tau)$ satisfies the modified Bessel equation

$$
\tau^{2} U^{\prime \prime}+\tau U^{\prime}+\left(-\tau^{2}-\gamma^{2}\right) U=0
$$

with $\gamma=\frac{1}{2} \sqrt{1-8 \lambda}$ and $\lambda<\frac{1}{8}$. The general solution can be written as a linear combination

$$
U(\tau)=\alpha I_{\gamma}(\tau)+\beta K_{\gamma}(\tau), \quad \alpha, \beta \in \mathbb{R}
$$

where $I_{\gamma}(\tau)$ and $K_{\gamma}(\tau)$ are the modified Bessel function of the first and second type. Hence the general solution of (4.18) is

$$
Z(\tau)=\sqrt{\tau} U(\tau)=\alpha \sqrt{\tau} I_{\gamma}(\tau)+\beta \sqrt{\tau} K_{\gamma}(\tau)
$$

where

$$
I_{\gamma}(\tau) \sim \sqrt{\frac{1}{2 \pi \tau}} e^{\tau}, \quad K_{\gamma}(\tau) \sim \sqrt{\frac{\pi}{2 \tau}} e^{-\tau} \text { as } \tau \rightarrow \infty
$$

see Haberman [3], p. 323.

Consequently, the solution of the extended transport equation (4.17) will be given by

$$
V\left(x_{0}, x, t\right)=t^{-\frac{1}{2}} Z(\tau)=\frac{\sqrt{x x_{0}}}{t}\left(\alpha I_{\gamma}\left(\frac{x x_{0}}{t}\right)+\beta K_{\gamma}\left(\frac{x x_{0}}{t}\right)\right),
$$

with $\alpha, \beta \in \mathbb{R}$.

\section{The heat kernel for $\mathbb{L}=\frac{1}{2} \partial_{x}^{2}+\frac{\lambda}{x^{2}}$}

In this section we shall state and prove the first main result of the paper.

Theorem 5.1. The heat kernel for the operator $\mathbb{L}=\frac{1}{2} \partial_{x}^{2}+\frac{\lambda}{x^{2}}$, with $\lambda<\frac{1}{8}$ and $x_{0}, x>0$ is

$$
\mathcal{K}\left(x_{0}, x ; t\right)=\frac{\sqrt{x_{0} x}}{t} I_{\gamma}\left(\frac{x_{0} x}{t}\right) e^{-\frac{x_{0}^{2}+x^{2}}{2 t}}, \quad t>0,
$$

where $I_{\gamma}$ is the nonsingular modified Bessel function of order $\gamma=\frac{1}{2} \sqrt{1-8 \lambda}$. 
Proof. We have shown already in the previous section that

$$
\left(\partial_{t}-\mathbb{L}\right) \mathcal{K}\left(x_{0}, x, t\right)=0, \quad t>0,
$$

with

$$
\mathcal{K}\left(x_{0}, x, t\right)=V\left(x_{0}, x, t\right) e^{-\frac{x_{0}^{2}+x^{2}}{2 t}},
$$

and $V$ given by (4.20). We need to choose the constants $\alpha$ and $\beta$ such that

$$
\lim _{t \searrow 0} \mathcal{K}\left(x_{0}, x, t\right)=\delta_{x_{0}}
$$

in the distributions sense. Let $K=K_{1}+K_{2}$ with

$$
\begin{aligned}
& \mathcal{K}_{1}\left(x_{0}, x, t\right)=\alpha \frac{\sqrt{x x_{0}}}{t} I_{\gamma}\left(\frac{x x_{0}}{t}\right) e^{-\frac{x^{2}+x_{0}^{2}}{2 t}} \\
& \mathcal{K}_{2}\left(x_{0}, x, t\right)=\beta \frac{\sqrt{x x_{0}}}{t} K_{\gamma}\left(\frac{x x_{0}}{t}\right) e^{-\frac{x^{2}+x_{0}^{2}}{2 t}} .
\end{aligned}
$$

Then

$$
\begin{aligned}
\lim _{t \searrow 0} \mathcal{K}_{1}\left(x_{0}, x, t\right) & =\lim _{t \searrow 0} \alpha \frac{\sqrt{x x_{0}}}{t} I_{\gamma}\left(\frac{x x_{0}}{t}\right) e^{-\frac{x x_{0}}{t}} e^{-\frac{\left(x-x_{0}\right)^{2}}{2 t}} \\
& =\alpha \lim _{\tau \rightarrow \infty} \sqrt{\tau} I_{\gamma}(\tau) e^{-\tau} \lim _{t \searrow 0} \frac{1}{\sqrt{t}} e^{-\frac{\left(x-x_{0}\right)^{2}}{2 t}} \\
& =\alpha \frac{1}{\sqrt{2 \pi}} \lim _{t \searrow 0} \frac{1}{\sqrt{t}} e^{-\frac{\left(x-x_{0}\right)^{2}}{2 t}} \\
& =\alpha \delta_{x_{0}},
\end{aligned}
$$

where we have used the first relation of (4.19). Hence we shall choose $\alpha=1$.

A similar computation, using the second relation of (4.19) yields

$$
\begin{aligned}
\lim _{t \searrow 0} \mathcal{K}_{2}\left(x_{0}, x, t\right) & =\lim _{t \searrow 0} \beta \frac{\sqrt{x x_{0}}}{t} K_{\gamma}\left(\frac{x x_{0}}{t}\right) e^{-\frac{x x_{0}}{t}} e^{-\frac{\left(x-x_{0}\right)^{2}}{2 t}} \\
& =\beta \lim _{\tau \rightarrow \infty} \sqrt{\tau} K_{\gamma}(\tau) e^{-\tau} \lim _{t \searrow 0} \frac{1}{\sqrt{t}} e^{-\frac{\left(x-x_{0}\right)^{2}}{2 t}} \\
& =\beta \sqrt{\pi} \lim _{\tau \rightarrow \infty} e^{-2 \tau} \lim _{t \searrow 0} \frac{1}{\sqrt{2 \pi t}} e^{-\frac{\left(x-x_{0}\right)^{2}}{2 t}} \\
& =0 .
\end{aligned}
$$

Hence

$$
\lim _{t \searrow 0} \mathcal{K}\left(x_{0}, x, t\right)=\lim _{t \searrow 0} \mathcal{K}_{1}\left(x_{0}, x, t\right)+\lim _{t \searrow 0} \mathcal{K}_{2}\left(x_{0}, x, t\right)=\alpha \delta_{x_{0}}
$$


so we need to choose $\alpha=1$. In order to find $\beta$ we shall consider the limit $\lambda \rightarrow 0$, case in which we recover the Gaussian kernel

$$
\begin{aligned}
\frac{1}{\sqrt{2 \pi t} e^{-\frac{\left(x-x_{0}\right)^{2}}{2 t}}} & =\lim _{g \searrow 0} \mathcal{K}\left(x_{0}, x, t\right) \\
& =\frac{1}{\sqrt{t}} \sqrt{\tau} I_{\frac{1}{2}}(\tau) e^{-\tau} e^{-\frac{\left(x-x_{0}\right)^{2}}{2 t}}+\beta \frac{1}{\sqrt{t}} \sqrt{\tau} J_{\frac{1}{2}}(\tau) e^{-\tau} e^{-\frac{\left(x-x_{0}\right)^{2}}{2 t}} \\
& =\frac{1}{\sqrt{2 \pi t}} e^{-\frac{\left(x-x_{0}\right)^{2}}{2 t}}+\beta \frac{1}{\sqrt{t}} \sqrt{\tau} J_{\frac{1}{2}}(\tau) e^{-\tau} e^{-\frac{\left(x-x_{0}\right)^{2}}{2 t}}
\end{aligned}
$$

since we take $\gamma=\frac{1}{2}$ in

$$
I_{\gamma}(\tau)=\frac{1}{\sqrt{2 \pi \tau}} e^{\tau-\frac{\frac{1}{2}\left(\gamma^{2}-\frac{1}{4}\right)}{\tau}+\mathcal{O}\left(\tau^{-2}\right)} .
$$

Hence we need to choose $\beta=0$. Then (4.20) becomes

$$
V\left(x_{0}, x, t\right)=\frac{\sqrt{x x_{0}}}{t} I_{\gamma}\left(\frac{x x_{0}}{t}\right)
$$

and hence (5.22) provides the heat kernel (5.21).

Remark 5.2. Since $I_{\gamma}>0$ it follows that the heat kernel $K\left(x_{0}, x ; t\right)>0$ for $t>0$.

\section{The two dimensional case}

We shall extend the above results in the case of two variables, where the operator becomes

$$
\mathbb{L}=\frac{1}{2}\left(\partial_{x_{1}}^{2}+\partial_{x_{2}}^{2}\right)-\frac{\lambda^{2}}{x_{1}^{2}+x_{2}^{2}}
$$

We note that in this case the constant $\lambda^{2} \geq 0$. In order to find the heat kernel of $\mathbb{L}$ we shall recall first a few basic properties of special functions.

The Laguerre polynomial $L_{n}^{a}$ of degree $n$ and parameter $a$ can be defined either by the Rodrigues' formula

$$
L_{n}^{a}=\frac{e^{x} x^{-a}}{n !} \frac{d^{n}}{d x^{n}}\left(e^{-x} x^{n+a}\right)=\frac{1}{n !}(-x)^{n}+\ldots,
$$


or equivalently, by the generating function formula

$$
\sum_{n \geq 0} L_{n}^{a}(x) y^{n}=\frac{1}{(1-y)^{a+1}} e^{x y /(y-1)} .
$$

Applying integration by parts several times yields

$$
\int_{0}^{\infty} L_{n}^{a}(x) L_{m}^{a}(x) e^{-x} x^{a} d x= \begin{cases}0, & \text { if } n \neq m \\ \frac{\Gamma(a+n+1)}{n !}, & \text { if } n=m\end{cases}
$$

Hence

$$
f_{n}(x)=\sqrt{\frac{n !}{\Gamma(a+n+1)}} e^{-x / 2} x^{a / 2} L_{n}^{a}(x), \quad n=0,1, \ldots
$$

form an orthogonal system for $\mathfrak{L}^{2}(0, \infty)$. One can show that this system is also complete. Using that $y=L_{n}^{a}(x)$ verifies the Laguerre equation equation

$$
x y^{\prime \prime}+(\alpha+a-x) y^{\prime}+n y=0,
$$

one can show that the functions (6.24) are eigenfunctions for the operator

$$
\mathbb{L}=x \partial_{x}^{2}+\partial_{x}-\frac{1}{4}\left(x+\frac{a^{2}}{x}\right),
$$

with the corresponding eigenvalue $\lambda_{n}=-\left(n+\frac{\alpha+1}{2}\right)$.

The following result is known under the name of Hille-Hardy's formula, see Erdelyi [2], p. 189:

If $L_{n}^{a}$ denotes the Laguerre polynomial, and $I_{a}$ is the modified Bessel function, then for $|z|<1$ we have

$$
\sum_{n=0}^{\infty} \frac{n !}{\Gamma(n+a+1)} z^{n}(x y z)^{a / 2} L_{n}^{a}(x) L_{n}^{a}(y)=\frac{1}{(1-z)} e^{-\frac{(x+y) z}{(1-z)}} I_{a}\left(\frac{2 \sqrt{x y z}}{1-z}\right) .
$$

Proposition 6.1. Let $a, b \in \mathbb{R}$. The heat kernel of the operator

$$
\mathbb{L}_{b}=x \partial_{x}^{2}+\partial_{x}-\frac{1}{4}\left(b^{2} x+\frac{a^{2}}{x}\right)
$$


is given by

$$
\mathcal{K}_{b}\left(x_{0}, x, t\right)=\frac{b / 2}{\sinh (b t / 2)} e^{-\frac{b}{2}\left(x_{0}+x\right) \operatorname{coth}(b t / 2)} I_{a}\left(\frac{b \sqrt{x_{0} x}}{\sinh (b t / 2)}\right), \quad t>0 .
$$

Proof. We shall first find the heat kernel of the operator

$$
\mathbb{L}_{1}=x \partial_{x}^{2}+\partial_{x}-\frac{1}{4}\left(x+\frac{a^{2}}{x}\right) .
$$

Then using the homogeneity property we shall determine the heat kernel for $\mathbb{L}_{b}$.

In the following we shall let $z=e^{-t}$ and use that

$$
\frac{\sqrt{z}}{1-z}=\frac{1}{2 \sinh (t / 2)}, \quad 1+\frac{2 z}{1-z}=\frac{1+e^{-t}}{1-e^{-t}}=\operatorname{coth}(t / 2)
$$

Then the heat kernel for the operator $\mathbb{L}_{1}$ on $(0, \infty)$ is given by the following expansion over the eigenfunctions (6.24)

$$
\begin{aligned}
\mathcal{K}_{1}\left(x_{0}, x, t\right) & =\sum_{n \geq 0} e^{\lambda_{n} t} f_{n}\left(x_{0}\right) f_{n}(x) \\
& =\sum_{n \geq 0} e^{-n t} e^{-\frac{a}{2} t} e^{-\frac{t}{2}} \frac{n !}{\Gamma(a+n+1)} L_{n}^{a}\left(x_{0}\right) L_{n}^{a}(x) x_{0}^{a / 2} e^{-x_{0} / 2} x^{a / 2} e^{-x / 2} \\
& =e^{-\frac{1}{2}\left(x_{0}+x\right)} \sqrt{z} \sum_{n \geq 0} \frac{n !}{\Gamma(a+n+1)} z^{n}\left(x_{0} x z\right)^{a / 2} L_{n}^{a}\left(x_{0}\right) L_{n}^{a}(x) \\
& =e^{-\frac{1}{2}\left(x_{0}+x\right)} \frac{\sqrt{z}}{1-z} e^{-\left(x_{0}+x\right) \frac{z}{1-z}} I_{a}\left(2 \sqrt{x_{0} x} \frac{\sqrt{z}}{1-z}\right) \quad \text { (by Hille-Hardy's formula) } \\
& =\frac{1}{2 \sinh (t / 2)} e^{-\frac{1}{2}\left(x_{0}+x\right)\left(1+\frac{2 z}{1-z}\right)} I_{a}\left(\sqrt{x_{0} x} \frac{1}{\sinh (t / 2)}\right) \\
& =\frac{1}{2 \sinh (t / 2)} e^{-\frac{1}{2}\left(x_{0}+x\right) \operatorname{coth}(t / 2)} I_{a}\left(\frac{\sqrt{x_{0} x}}{\sinh (t / 2)}\right) .
\end{aligned}
$$

In order to compute the heat kernel of the operator $\mathbb{L}_{b}$, we take $\tilde{x}=b x$ as a new variable. A computation shows $\mathbb{L}_{b}=b \tilde{\mathbb{L}}_{1}$. Then

$$
\left(e^{t \mathbb{L}_{b}} f\right)\left(x_{0}\right)=\left(e^{t b \tilde{\mathbb{L}}_{1}} \tilde{f}\right)\left(\tilde{x}_{0}\right)=\int K_{1}\left(b x_{0}, b x, b t\right) f\left(x_{0}\right) b d x_{0},
$$

and hence the heat kernel of $e^{t \mathbb{L}_{b}}$ is $b K_{1}\left(t x_{0}, t x, t b\right)$, that is

$$
\mathcal{K}_{b}\left(x_{0}, x, t\right)=\frac{b / 2}{\sinh (b t / 2)} e^{-\frac{b}{2}\left(x_{0}+x\right) \operatorname{coth}(b t / 2)} I_{a}\left(\frac{b \sqrt{x_{0} x}}{\sinh (b t / 2)}\right) .
$$

Therefore the heat kernel of the operator $\mathbb{L}_{b}$ is given by formula (6.27). 
Corollary 6.2. The heat kernel for $\mathbb{L}_{0}=x \partial_{x}^{2}+\partial_{x}-\frac{a^{2}}{4 x}$ is given by

$$
\mathcal{K}_{0}\left(x_{0}, x, t\right)=\frac{1}{t} e^{-\frac{1}{t}\left(x_{0}+x\right)} I_{a}\left(\frac{2 \sqrt{x_{0} x}}{t}\right), \quad t>0 .
$$

Proof. The heat kernel of $\mathbb{L}_{0}$ is obtained making $b \rightarrow 0$ in the relation (6.27). Using $\lim _{b \rightarrow 0} \frac{b t / 2}{\sinh (b t / 2)}=1$ yields (6.28).

Proposition 6.3. The heat kernel of $\mathbb{P}=\frac{1}{2}\left(\partial_{r}^{2}+\frac{1}{r} \partial_{r}-\frac{a^{2}}{r^{2}}\right)$ is given by

$$
G\left(r_{0}, r, \tau\right)=\frac{1}{2 \tau} e^{-\frac{1}{2 \tau}\left(r_{0}^{2}+r^{2}\right)} I_{a}\left(\frac{r_{0} r}{\tau}\right), \quad \tau>0 .
$$

Proof. Changing of variable $x=r^{2}$ in $\mathbb{L}_{0}$ yields

$$
\mathbb{L}_{0}=x \partial_{x}^{2}+\partial_{x}-\frac{a^{2}}{4 x}=\frac{1}{4}\left(\partial_{r}^{2}+\frac{1}{r} \partial_{r}-\frac{a^{2}}{r^{2}}\right) .
$$

Let $\tau=t / 2$. Since the following relation holds among the heat kernels

$$
e^{t\left(x \partial_{x}^{2}+\partial_{x}-\frac{a^{2}}{4 x}\right)}=e^{\frac{t}{4}\left(\partial_{r}^{2}+\frac{1}{r} \partial_{r}-\frac{a^{2}}{r^{2}}\right)}=e^{\frac{\tau}{2}\left(\partial_{r}^{2}+\frac{1}{r} \partial_{r}-\frac{a^{2}}{r^{2}}\right)},
$$

then the heat kernel of $\frac{1}{2}\left(\partial_{r}^{2}+\frac{1}{r} \partial_{r}-\frac{a^{2}}{r^{2}}\right)$ is obtained from the heat kernel of $x \partial_{x}^{2}+\partial_{x}-\frac{a^{2}}{4 x}$ by making the substitutions $x_{0}=r_{0}^{2}, x=r^{2}$ and $\tau=t / 2$ in formula (6.28).

As an application of formula (6.29) we shall find the heat kernel of the operator

$$
\mathbb{L}=\frac{1}{2}\left(\partial_{x_{1}}^{2}+\partial_{x_{2}}^{2}\right)-\frac{\lambda^{2}}{x_{1}^{2}+x_{2}^{2}},
$$

which in polar coordinates becomes

$$
\mathbb{L}=\frac{1}{2}\left(\partial_{r}^{2}+\frac{1}{r} \partial_{r}+\frac{1}{r^{2}} \partial_{\theta}^{2}\right)-\frac{\lambda^{2}}{r^{2}} .
$$

If $\mathcal{K}$ is the heat kernel of the above operator, then applying a partial Fourier transform with respect to $\theta$, we obtain that $\hat{\mathcal{K}}=\mathfrak{F}_{\theta} \mathcal{K}$ is the heat kernel of the operator

$$
\mathbb{P}=\frac{1}{2}\left(\partial_{r}^{2}+\frac{1}{r} \partial_{r}-\frac{1}{r^{2}} \xi^{2}\right)-\frac{2 \lambda^{2}}{2 r^{2}}=\frac{1}{2}\left(\partial_{r}^{2}+\frac{1}{r} \partial_{r}-\frac{a^{2}}{r^{2}}\right),
$$


with $a^{2}=2 \lambda^{2}+\xi^{2}$. The heat kernel of $e^{t \mathbb{P}}$ can be obtained from formula (6.29)

$$
\hat{\mathcal{K}}\left(r_{0}, r, \tau ; \xi\right)=\frac{1}{2 \tau} e^{-\frac{1}{2 \tau}\left(r_{0}^{2}+r^{2}\right)} I_{a}\left(\frac{r_{0} r}{\tau}\right) .
$$

Applying the inverse Fourier transform yields the heat kernel of the operator (6.30)

$$
\begin{aligned}
\mathcal{K}\left(r_{0}, \theta_{0}, r, \theta, \tau\right) & =\frac{1}{2 \pi} \int e^{i\left(\theta-\theta_{0}\right) \xi} \frac{1}{2 \tau} e^{-\frac{1}{2 \tau}\left(r_{0}^{2}+r^{2}\right)} I_{\left(2 \lambda^{2}+\xi^{2}\right)^{\frac{1}{2}}}\left(\frac{r_{0} r}{\tau}\right) d \xi \\
& =\frac{1}{4 \pi \tau} e^{-\frac{1}{2 \tau}\left(r_{0}^{2}+r^{2}\right)} \int e^{i\left(\theta-\theta_{0}\right) \xi} I_{\left(2 \lambda^{2}+\xi^{2}\right)^{\frac{1}{2}}}\left(\frac{r_{0} r}{\tau}\right) d \xi .
\end{aligned}
$$

To conclude, going back to the variables $x_{0}$ and $x$, we obtain:

Proposition 6.4. The heat kernel of the operator (6.30) is given by

$$
\mathcal{K}\left(x_{0}, x, \tau\right)=\frac{1}{4 \pi \tau} e^{-\frac{1}{2 \tau}\left(\left|x_{0}\right|^{2}+|x|^{2}\right)} V\left(\arg \left(x_{0}, x\right), \frac{x_{0} x}{\tau}\right), \quad \tau>0,
$$

where $\arg \left(x_{0}, x\right)=\cos ^{-1}\left(\frac{x \cdot x_{0}}{|x|\left|x_{0}\right|}\right)$, and

$$
V(u, \rho)=\int e^{i u \xi} I_{\left(2 \lambda^{2}+\xi^{2}\right)^{\frac{1}{2}}}(\rho) d \xi
$$

\section{Directions to further studies}

We suggest here a few open problems related to this paper. We first ask how can the method be extended to find the heat kernel for an elliptic operator with potential

$$
\begin{aligned}
\left(\frac{\partial}{\partial t}-\sum_{i, j=1} a_{i j}(x) \partial_{x_{i}} \partial_{x_{j}}-\frac{\lambda^{2}}{\sum a_{i j}(x) x_{i} x_{j}}\right) \mathcal{K}\left(x_{0}, x, t\right) & =0, \quad t>0 \\
\lim _{t \searrow 0} \mathcal{K}\left(x_{0}, x, t\right) & =\delta_{x_{0}} .
\end{aligned}
$$

In particular, we are interested in the role played by the geometry. Can we find the heat kernel for $\mathbb{L}=\frac{1}{2} \sum \partial_{x_{k}}^{2}-\frac{\lambda^{2}}{|x|^{2}}$, with $\lambda \neq 0$ and $x \in \mathbb{R}^{n}$ with $n \geq 3$ ? 


\section{References}

[1] Calin, O., Chang, D. C., Geometric Mechanics on Riemannian Manifolds, Birkhäuser, (2004).

[2] Erdélyi, A., Higher Transcendental Functions, McGraw-Hill, vol. 2, (1953).

[3] Haberman, R., Elementary Applied Partial Differential Equations, 3-rd ed., Prentice Hall, (1988).

[4] Khandekar, D.C., Lawande, S. V., Path integration of a three body problem, J. Phys. A: Gen. Phys., Vol. 5, (1972).

[5] Khandekar, D.C., Lawande, S. V., Exact propagator for a time dependent harmonic oscillator with and without a singular perturbation, J. Math. Phys., Vol. 16, No. 2, (1975).

[6] Schulman, L. S., Techniques and Applications of Path Integration, Dover, (2005).

Ovidiu Calin

Department of Mathematics

Eastern Michigan University,

Ypsilanti, MI, 48197, USA

E-mail: ocalin@emich.edu

Der-Chen Chang

Department of Mathematics

Georgetown University,

Washington DC 20057, USA

E-mail: chang@math.georgetown.edu 